\title{
Evaluation of Inter-Cell Interference Coordination with CAP model
}

ydxu@tsinghua.edu.cn

\author{
Yida $\mathrm{Xu}^{1}$, Zhigang Tian ${ }^{1}$, Wenqi Wang ${ }^{1}$, Ming \\ $\mathrm{Zhao}^{1}$, Xibin $\mathrm{Xu}^{1}$, Jing Wang ${ }^{1}$, Wei(Andrew) Tan ${ }^{2}$ \\ 1. Tsinghua National Laboratory for Information \\ Science and Technology, Tsinghua University \\ Beijing, China \\ 2. HUAWEI Technology Co., Ltd \\ Shanghai, China
}

\author{
Shih-Chang Chen ${ }^{3}$, Yeh-Ching Chung ${ }^{3}$ \\ 3. Department of Computer Science \\ National Tsing Hua University \\ Taipei, Taiwan \\ ychung@cs.nthu.edu.tw
}

\begin{abstract}
More cooperation or coordination is necessary for future UDN (Ultra-Dense Network) scenario of 5G network, of which ICIC (Inter-Cell Interference Coordination) is a promising and typical scheme. Cooperating cellular network may be thought as a special kind of distributed computing system, whose basic theory and toolsets could be adopted. CAP theory for distributed computing state that any networked shared-data system can have at most two of three desirable properties: consistency (C), high availability (A) and tolerance to network partitions $(P)$ of the data. Since partition could not be avoided in such system, consistency or availability has to be forfeited to a certain extent. This paper extends the CAP theorem to the UDN case, and redefines the consistency, availability and partitiontolerance in the context of future cellular network architecture. Then ICIC process are interpreted with cellular CAP theorem, and the evaluation framework is developed. An improved ICIC scheme is proposed based on such framework.
\end{abstract}

\section{Keywords-UDN, ICIC, CAP, BASE, Distributed Computing}

\section{INTRODUCTION}

Mobile data traffic volume has entered into a rapid growing period for at least 7 years according to Cisco VNI [1]. The annual forecast and real recap are shown in Figure 1, CAGR prediction is adjusted lower for each year in a reasonable style; practical CAGR is also slow down after the exploding between 2019 and 2011; the curve of the practical volume between 2008 and 2014 is joined by the curve of predicted volume between 2014 and 2019. Although the growth is lowered to a reasonable speed, the overall volume is still following an exponential curve. The optimist affirms that the increasing investment on network is always able to meet the requirement. However, the telecommunication industry has to think about the possible technical solutions and the impact of growing traffic volume.

The story of growing mobile traffic is not new, since it has been summarized by Cooper's Law [2]. The Law states that the maximum number of voice conversations or equivalent data transactions that can be conducted in all of the useful radio spectrum over a given area doubles every 30 months. The Law keeps for past 104 years, and the effectiveness of personal communications total spectrum utilization is improved about 1 million times in the last 45 years. The overall improvement is decoupled into 25 times improvement of spectrum efficiency, 25 times of spectrum deployment, and 1600 times increasing of network density.

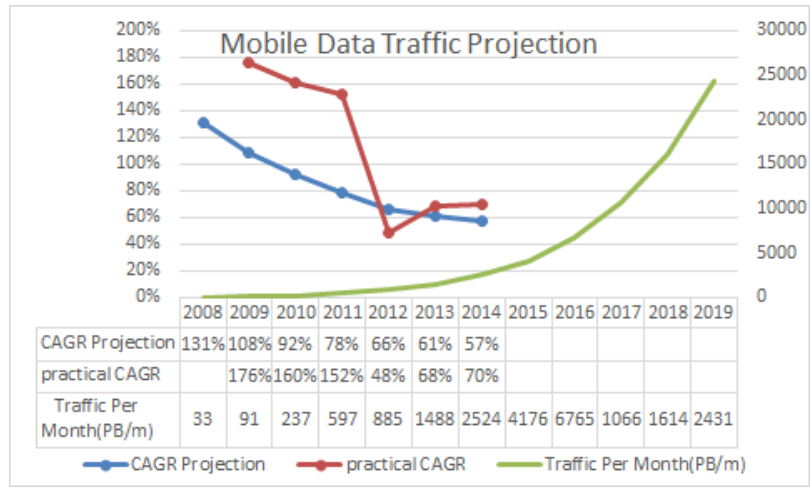

Figure 1 Growth of Mobile Data Traffic

Mobile telecommunication industry has been using above technical combination to meet growing requirement, and develop from $1 \mathrm{G}$ of 30 years ago to $4 \mathrm{G}$ of today. Cooper's Law may keep true for future $5 \mathrm{G}$ and late, however the technical solutions behind cooper's law would change. 3GPP held a special workshop to discuss the possible technologies for the mobile data traffic of 2020 era [3]. Since there is little room for the improvement of pure transmission spectrum efficiency, the following technologies combination is proposed: 3 times of spectrum employed, 6 times of spectrum efficiency and 56 times of network density.

The speed of network density would be accelerated, which means the cell radius would decrease from 4G (LTE)'s $500 \mathrm{~m}$ to about $50 \mathrm{~m}$. In such Ultra Dense Network (UDN) scenario, current cellular networking model may change with the increasing inter-cell interferences, frequent handover, etc.. Based on some advanced technologies, 3GPP developed a lot of schemes to cope with these changes, include Coordinated multipoint (CoMP) transmission and reception, ICIC [4], eICIC [5], small cell enhancement [6], etc.. All these schemes need tight cooperation or coordination between different cells or BBUs, and 3GPP defines the X2 interface for such inter-cell cooperation [6]. However the development of X2 interfaces lags behind Uu interface, which may block the adoption of 
above technologies in practical network. A cooperation model for emerging cellular networking should be developed, based on which the behavior between cells or base stations could be studied. Such a general cooperation framework and interactive mechanism may be specified for X2 interface, which would facilitate the development and deployment of innovational cooperation schemes. In the following parts of this paper, such framework would be elaborated with ICIC as objective.

\section{INTER-CELL INTERFERENCE COORDINATION}

ICIC is a kind of efficient technology to migrate the intercell interference for OFDM system [8]. 3GPP develop a serial of special schemes for LTE system from R8 to R12 [4-5]. In R8/9, some basic ICIC schemes are specified for homogeneous cellular network, including Partial Frequency Reuse (PFR) and Soft Frequency Reuse (SFR). R10 introduce Carrier Aggregation (CA) and Heterogeneous networks (HetNet), so corresponding ICIC schemes are also improved as CA enhanced ICIC, and non-CA ABS (Almost Blank Subframes) based eICIC. Actually ICIC is the hot topic in 3GPP, so a lot of contribution propose different innovational variants for above schemes.

Basically, ICIC is a kind of radio resource scheduling schemes. Therefore frequency, time and power resources could be adjusted or scheduled for avoiding the inter-cell interferences. Both PFR and SFR could be combined with Power Control, and ABS schemes schedule the transmission opportunities in time domain. ICIC schemes could be categorized into centralized control and distributed coordination. Since LTE network architecture is lack of the centralized control point like RNC (Radio Network Controller) of $3 \mathrm{G}$, most of ICIC schemes in LTE belongs to the second kind. According to the frequency of radio resources adjustment, ICIC schemes could be characterized into static, semi-static and dynamic style.

LTE system adopts a flat network architecture without centralized control point, which is suitable for distributed ICICs. However symmetric eNodeBs may choose one master to control other slaves in one coordinating sets. Therefore we would not emphasis the difference between centralized coordination and distributed coordination. We will elaborate the static, semi-static and dynamic ICIC in this sections. Static ICIC is the most simple and still promising schemes, which may be inferior to the other schemes in performance but superior in complexity. Static ICIC can be viewed as the basic building block for other advance schemes, so only two basic static ICIC schemes are elaborated here. Other semi-static and dynamic schemes are derived from the basic ideas.

\section{A. PFR}

In PER, the frequency sub-bands are allocated in static frequency planning without adjustment according to variance of traffic, load and interference condition. Two group of subbands are allocated statically: Cell-Center Area (CCA) and Cell-Edge Area (CEA). Since CCA has lower interference level then higher SINR (Signal to Interference and Noise Ratio), low reuse factor (e.g., 1) could be adopted to achieve better spectral efficiency. In CEA region, higher reuse factor is used to avoid severe interference level from neighbor cells. PER is simple in its implementation complexity, while spectral efficiency is sacrificed with high reuse factor.
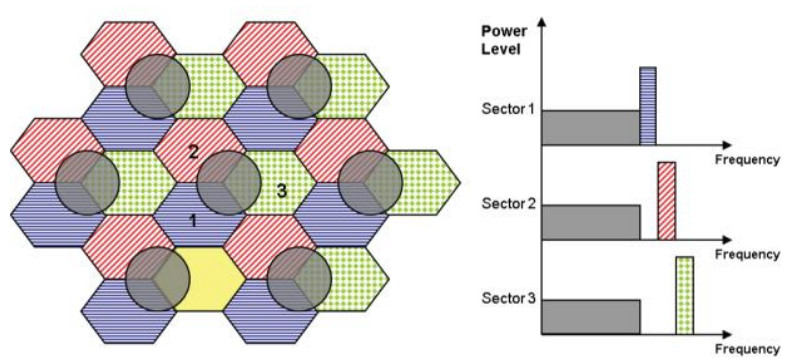

Figure 2 Partial Frequency Reuse [4]

\section{B. SFR}

Different from PFR, each cell could use the full bandwidth in SFR. The full band is divided into N sub-bands, while CCA users could use all the sub-bands of low power mode and CEA users use high power band with a certain of reuse factor. Furthermore, the unutilized sub-bands of CEA could also be used by CCA users since no more inter-cell interferences would be created. In Figure 3, reuse factor $\mathrm{N}=3$ is adopted for CEA.
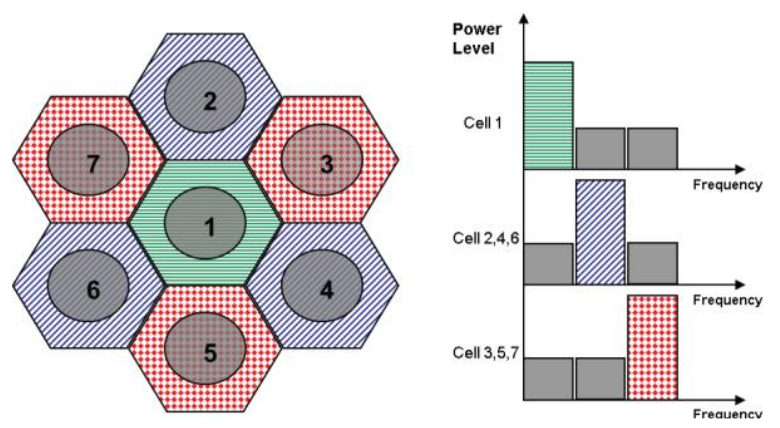

Figure 3 Soft Frequency Reuse N=3 [4]

For CCA user, reuse factor 1 is achieved with SFR scheme. While PFR is a more conservative scheme with exclusive allocation for CCA and CEA. Although SFR bring a lot of flexibility and performance improvement, it could not adaptive to the variance of environment and traffic load. Except for static frequency planning, no cooperation is needed on the fly.

\section{Semi-static ICIC and Dynamic ICIC}

Interference is mostly relative with the changing of traffic arrival/departure, load and model, which is in semi-static scale comparing to the rapid changing of wireless channel. Therefore the coordination in semi-static scale is effective to migrate the interference brought by traffic variance. The semi-static coordination leads to the semi-static signaling across X2 interface, which is an acceptable load for backhaul. Derived from static ICIC, both PFR and SFR could be modified as semi-static schemes.

Dynamic ICIC is more aggressive to cope with the rapid changing of wireless channel and environment. This kind of algorithms could approach the optimal performance (capacity or area spectral efficiency) with some sub-optimal methods, since joint scheduling for cellular network is a non-convex

This paper was supported in part by the MOST "Hong Kong, Macao and Taiwan" Science Collaboration Project (2014DFT10320); HUAWEI

"Research on Soft Defined Protocol" Project. 
optimization problem. Typical sub-optimal algorithms may be based on graph theory, game theory and heuristic solutions. The high implementation complexity of dynamic ICIC is one drawback, but the high signaling overhead across X2 interface in sub-frame or frame level is more important for the adoption of this kind of schemes. Despite the overhead, the latency requirement brought by dynamic coordination make it infeasible in most of practical cellular environment.

Based on above discussion, we would use only semi-static ICIC as the objective for the remaindering parts of this paper. Also, the overhead and latency of coordination signaling gives light on the adoption of CAP framework of distributed computing theory.

\section{ICIC IN CAP FRAMEWORK}

CAP theorem for distributed computing state that any networked shared-data system can have at most two of three desirable properties: consistency (C), high availability (A) and tolerance to network partitions $(\mathrm{P})$ of the data. Since partition could not be avoided in such system, consistency or availability has to be forfeited to a certain extent. The CAP theorem is brought by Eric Brewer in 2000 [9], Gilbert and Lynch [10] proved Brewer's conjecture about CAP theorem. CAP theorem meets or guides the development of NoSQL database and large scale web system [11]. Though it is described and discussed in web or database terminologies, the root if CAP model is in distributed computing system. Since the cellular networking in UDN scenario need tight cooperation between cells or base stations, the networking model is more like a distributed computing system. Furthermore, the implementation of such networking process may need the support of middleware or framework of distributed computing system. This paper would apply the CAP model in cellular networking, while the adoption of distributed computing system would be studied in the future.

\section{A. General CAP model}

CAP theorem is very popular in the design of NoSQL database, which is the core of scalable design of large scale distributed web system. Nathan summarized different NoSQL design and categorized them into CAP model in a visual guide [11]. The most popular interpretation of CAP theorem of 'Pick two of three' is shown in Figure 4.

NoSQL database is designed to scale out instead of scaling up for better performance in terms of handling big data. While conventional RDBMS (Relation Database Management System) like MySQL could guarantee CA in the same time, because there is NO Partition in single mainframe server; NoSQL systems must tolerate network partition, because they employ large amount of servers to speed up the process of data manipulation. Therefore, NoSQL databases has to pick either AP or CP from CAP. Applications integrated with NoSQL databases have to consider their services with AP or CP, which means data requested by users must be the identical or allows users read data without consistency temporarily. Former may be applications related to economic activities which require data consistency strictly while later can be social network applications allowing some users not reading the latest replies of posts temporarily.

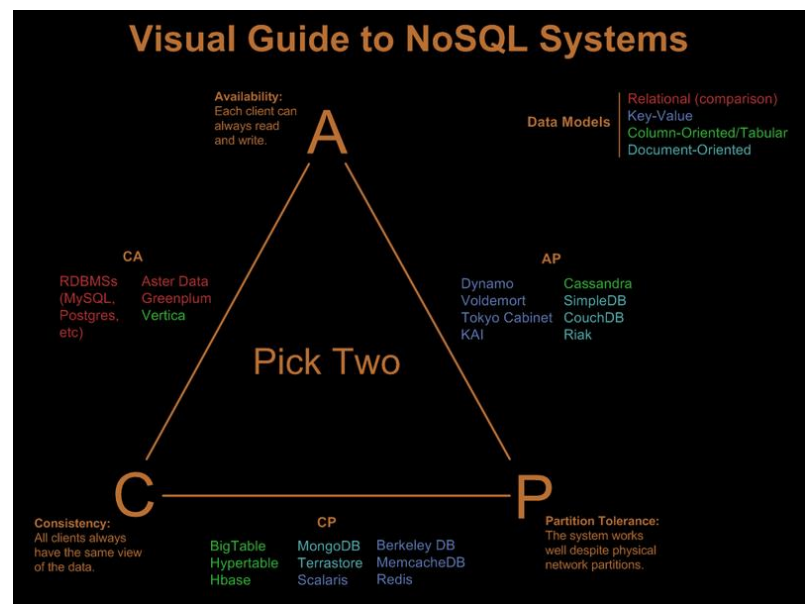

Figure 4 Visual Guide to NoSQL Systems on CAP Framework [11]

Although CAP theorem is prevailing in the design of large scale web system and NoSQL database. The meaning and metric of Consistency, Availability and Partition tolerance led to a lot of discussion and interpretation. The focus is on Partition Tolerance, because it's occasionally in current data center environment, both Consistent and Availability could be guaranteed perfectly. In another side, many issues like latency of network may cause some factual partition of network, so the designer has to consider $\mathrm{C}, \mathrm{A}$ or $\mathrm{P}$ in a certain scale. With such consideration, D.J. Abadi extend the CAP model as PACELC model [12]: if there is a partition $(P)$, how does the system trade off availability and consistency $(A$ and $C)$; else $(E)$, when the system is running normally in the absence of partitions, how does the system trade off latency $(L)$ and consistency $(C)$ ?

After twelve years of CAP theorem presented by Brewer, the interpretation and adoption of CAP model is approaching the unification. IEEE Computer Society publish a special issue on CAP theorem [13-14]. System designers should consider the trade-off between Consistency and Availability according to their services since Partition is unavoidable usually. Different management strategies can be developed for various issues which cause network partitions. Namely, if data inconsistency and unavailability are acceptable for a short period of time according to the SLA (service-level agreement) of their services, then a system may have properties of both $\mathrm{C}$ and $\mathrm{A}$ conditionally.

\section{B. Conventional Cellular Networking under CAP Model}

The behavior of conventional cellular networking is shown in Figure 5. The cells are almost independent in its resource allocation, however each cell would suffer the interferences from surrounding cells. Through the measurement report of the terminal, the cell obtains a certain information about surrounding cells. Imaging terminals do the calculation as the equation in Figure 5 and return the results to serving cell, by this means the other cells tell the serving cell the information of their resource allocation. Since above implicit interactive channel is so limit, the whole cellular networking would approach the stability state in an iterative form. With the changing of channel and traffic, the stability state may be 
broken and the cellular networking begin to seek for new stability state. The procedure is similar as gaming in game theory, and the stability state is similar as Nash Equilibrium.

Another cooperation mechanism in conventional cellular networking model is handover, two or more cells change the serving cell of some terminal based on measurement report, which need some interactive signaling across backhaul.

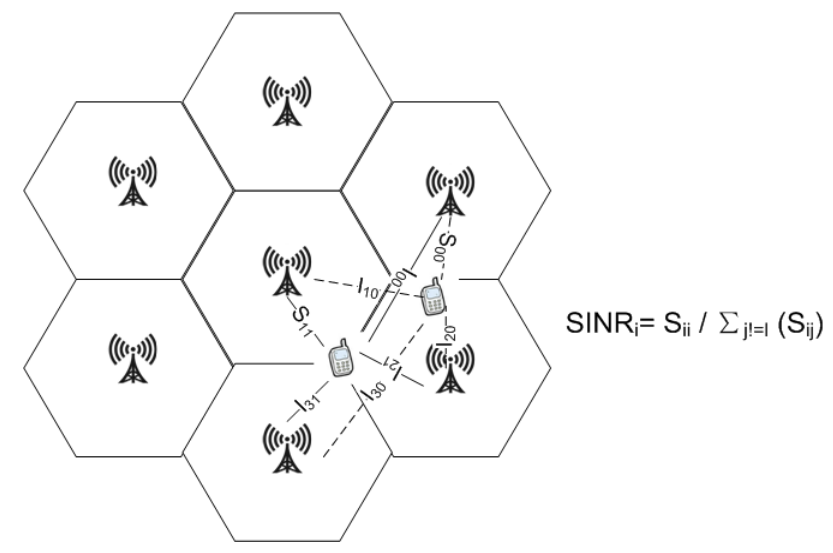

Figure 5 Conventional Cellular Networking Model

From above elaboration, it's observed that the cooperation between different cells is limit, which is suitable for the limit or expensive transmission capability of previous backhaul. Since the backhaul or fronthaul of modern cellular system is more powerful than $2 \mathrm{G} / 3 \mathrm{G}$ system, more cooperation is possible between different cells. Moreover, the collaboration in UDN scenario is necessary as described above. The cooperating cells or base stations could be thought as a distributed computing system. Therefore, the adoption of CAP model in cellular networking and the utilization of CAP model in the network architecture is reasonable.

\section{CAP Model for Modern Cellular Networking}

The cells could be thought as the computing nodes in distributed computing system, and the limit transmission capability or long latency between cells could be thought as a certain of Partition. Therefore conventional cellular network is severe Partitioned, Availability of service for terminals is preferred with $95 \%$ confidence. As the results, the Consistent has to be sacrificed to a certain, which means the biggest capability or best performance is always achieved in an iterative style. In other words, the consensus is approached eventually, which is stated as Eventually Consistent in CAP theorem. Above is the case for conventional cellular networking, while modern cellular networking need or have more coordination, the tradeoff of Consistent, Availability and Partition Tolerance could be dealt with in different extent.

As described above, the CAP model is extended for cellular networking. Before the detail description, a basic term-cell is reinterpreted. Conventional cell manipulates a bundle of radio resource (time, frequency and transmit power), which is reused between different cells. A cell is also the entity providing service (access, authentication and transmission) for some terminal, and usually the service is conveyed by a base station (combination of RRU and BBU) in network side. Since the interference exists for the transmission occupying the relevant radio resource between different cells, the coordination may be needed across relevant cells. The coordination may be executed by base station with the aid of fronthaul, backhaul or terminal feedback. Since the introducing of HetNet in LTE system, the definition of cell is different from conventional one. A cell may don't have complete radio resource set, and cannot provide full suite of service independently. The coverage of a cell may formed by a group of antennas, and the process of a cell may be conveyed in any infrastructure not bundled to a BBU. The coordination between cells may aim for interference avoidance, or cooperation to provide complete service for some terminal. Dual connectivity by macro cell and small cell could be thought as the example of later.

With the extended definition of cell, the CAP model could also be explained as:

- Partition is determined according to the transmission latency and capability of FrontHaul and BackHaul. If the latency/capability between two cells is large/lower than acceptable threshold, they are in different Partitions.

- Availability is measured as the opportunity of any terminal's request being severed with desired quality in any place and any time. Usually this property is in high priority to be satisfied, e.g., 95\% of terminal's request should be served with their desired QoS.

- Consistency level could be evaluated as how close the immediate performance is to the optimal performance or how long (many iterates) the optimal performance is achieved eventually. Actually we are discussing the equilibrium, however it could be viewed as the extent of consistency of practical performance to the optimal performance known in the global supervisor. The global supervisor know everything immediately but cannot feedback anything, so it's in an isolated Partition.

From above description, CAP model is extended for modern cellular network and three properties are redefined with some metrics. Therefore, these three properties could be traded off in a certain extent.

\section{Semi-static ICIC in extended CAP Model}

Since semi-static ICIC is promising in its' moderate complexity and performance, we use such scheme as objective to describe the tradeoff according to extended CAP model. With each cell as central point, the whole cell layout is separated as three tie:

- Tight Coordination Tie, the cells in which could be cooperated perfectly in one semi-static cycle without consideration of latency and capability of BackHaul and FrontHaul, e.g., a data center.

- Limit Coordination Tie, in which several coordination iterations may be executed between different coordination tie for a semi-static cycle.

- Gaming Tie, in which only the historical decision and feedback of previous semi-static cycle is available. 
Based on the observer on previous cycles, the decision is drawn for current cycle.

There is no distinct partition here, since three tie for different cells are overlapped. A possible procedure for semistatic ICIC is:

- Step 1: Collect the historical decision from other cells and the feedback from the terminals of Gaming Tie in previous semi-static cycles

- Step 2: Collect the decisions from other cells of Limit Coordination Tie in previous iteration of current semistatic cycle

- Step 3: Perform centralized or perfect distributed coordination within Tight Coordination Tie, with the consideration of the collected data in Step 1\&2

- $\quad$ Step 4: Return to Step 2 until the iteration number for current semi-static cycle is reached

- Step 5: Perform the allocation or adjustment for current semi-static cycle and proceed to Step 1 of next semistatic cycle

Based on above procedure, different strategies could be adopted to tradeoff Consistency, Availability and Partition. If Availability criterion is fixed, different Consistency level may be achieved with different Partition condition; If Partition condition is fixed, Consistency level may be improved with the sacrifice of Availability, e.g., prolonging response time to service request; If the desired Consistency level is mandatory, different Availability level (e.g., the response time) could be achieved in different Partition scenarios (e.g., hotspot and roadside).

\section{NETWORK ARCHITECTURE AND CAP MODEL}

Network Architecture is related with CAP model in two ways:

- A general network architecture should be given as the base of elaboration of CAP framework for UDN scenario, and the architecture need define the cooperating entity and mechanism.

- Consider which tools or framework related with current CAP theorem could be adopted in such network architecture.

We have organized the whole mobile network into 4 domains and 4 layers. For different cross point of domain and layer, corresponding schemes can be developed with the same goal of open network architecture:

- Open Operation \& Business : Networking and Operation as a Service

- Open Cellular Networking: HCA (Hyper Cellular Architecture)

- Open FrontHaul: Coverage Subsystem

- Open Infrastructure: YaRAN ( Yet another RAN)

- Open Network Protocol: NoStack (Not only Stack)
- Open Terminal Architecture: Nostack4UE \& YaRAN4UE

- Open Air Interface Waveform

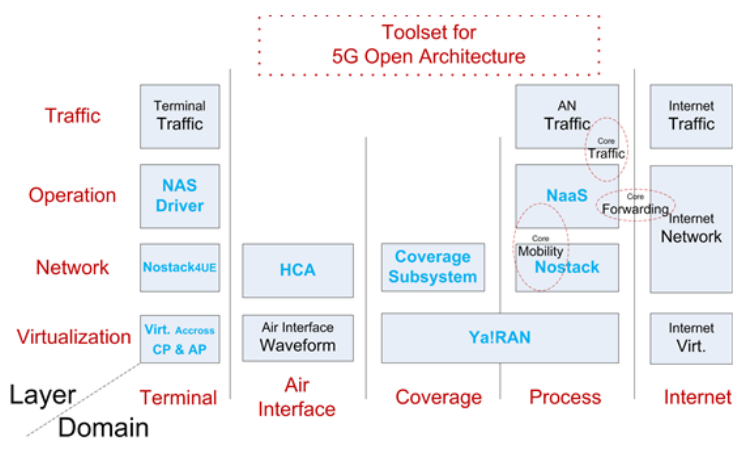

Figure 6 Framework for 5G Network Architecture

Here, our focuses are HCA, YaRAN, Coverage Subsystem and NoStack. These technical solutions are mapped in to a big picture as below figure.

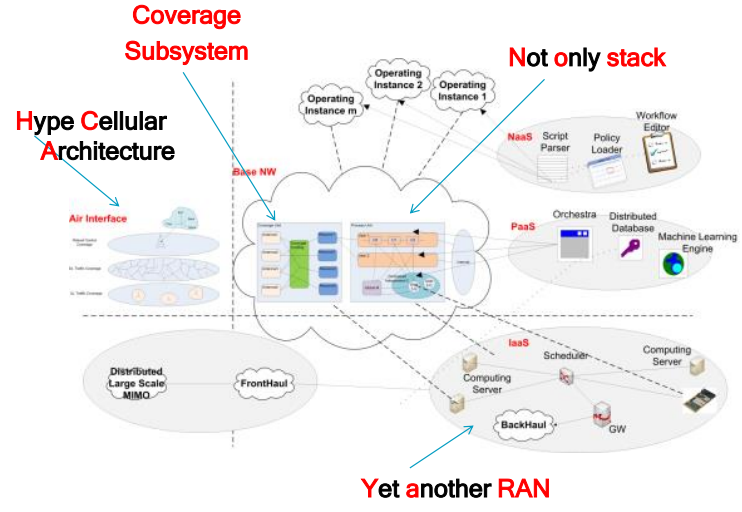

Figure 7 5G Open Architecture

The characteristics of HCA is the decoupling of different type of coverage, e.g., control cell, traffic cell and sniffer cell for RACH may be different. YaRAN is the infrastructure and related virtualization platform for RAN, which include FrontHaul, BackHaul and data center for BBU cloud. NoStack decouples the network process into different flows, e.g., the flow for user oriented cell and the flow for control cell. The responsibility of Coverage Subsystem is mapping between the $\mathrm{Tx} / \mathrm{Rx}$ services of antennae and requirement of each flow. Coverage Subsystem is also controlled by NoStack. For the elaboration of CAP model and ICIC like algorithms, Coverage Subsystem in Figure 8 is the key point to decouple the coverage and process. Therefore the cooperation between cells could be transformed as that between antennae.

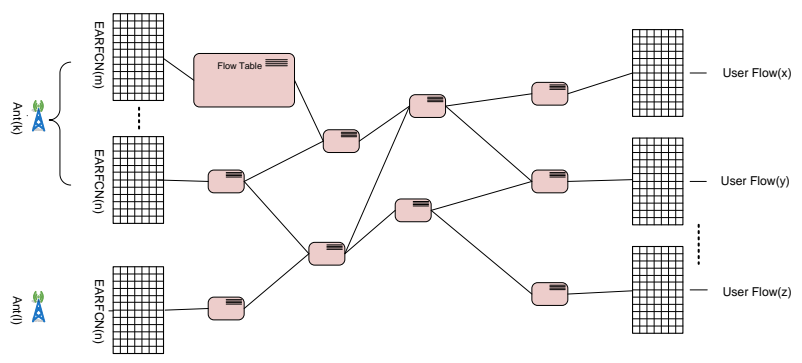

Figure 8 Coverage Subsystem to decouple coverage and process 


\section{CONCLUSION AND FUTURE WORK}

In this paper, the CAP theorem from distributed computing system is brought to the cellular networking in UDN scenario. The CAP framework is interpreted in the context of cellular networking, and the cooperation of cellular network is explained with extended CAP framework. Semi-static ICIC schemes is chosen as the objective for such interpretation.

In future work, the role of Coverage Subsystem in the semistatic ICIC or other coordination schemes should be studied. The evaluation method should be given for the extended CAP model and the benchmarking (simulation) should be performed according to such model. And, suitable tools or framework to implementation of the network architecture in UDN scenario should be chosen from current distributed computing toolsets, or developed according to the principle of extended CAP model.

\section{REFERENCES}

[1] Cisco System, "Cisco Visual Networking Index: Global Mobile Data Traffic Forecast Update 2014-2019", January, 2015.

[2] Martin Cooper , "Cooper's Law", http://www.arraycomm.com/ technology/coopers-law/

[3] 3GPP RAN, "Future Radio in 3GPP", http://www.3gpp.org /news-events/3gpp-news/1266-Future-Radio-in-3GPP, June 2012.
[4] Emmanouil Pateromichelakis, Mehrdad Shariat, Atta ul Quddus, and Rahim Tafazolli, "On the Evolution of Multi-Cell Scheduling in 3GPP LTE / LTE-A", IEEE COMMUNICATIONS SURVEYS \& TUTORIALS, VOL. 15, NO. 2, SECOND QUARTER 2013.

[5] D. Lopez-Perez, I. Guvenc, G. de la Roche, M. Kountouris, T. Quek, and J. Zhang, "Enhanced intercell interference coordination challenges in heterogeneous networks," IEEE Wireless Commun., vol. 18, no. 3, pp. 22 -30, June 2011.

[6] 3GPP TR 36.932, "Scenarios and requirements for small cell enhancements for E-UTRA and E-UTRAN (Release 12)", March 2013.

[7] 3GPP TS 36.423, "X2 Application Protocol (X2AP)", March 2015.

[8] A. Hamza, S. Khalifa, H. Hamza and K. Elsayed, "A survey on inter-cell interference coordination techniques in OFDMA-based cellular networks", IEEE Commun. Surveys Tuts., vol. 15, no. 4, 2013.

[9] E. Brewer, "Towards Robust Distributed Systems," in Principles Of Distributed Computing, Portland, 2000. [Online]. Available: www.cs.berkeley.edu/ brewer/cs262b-2004/PODC-keynote.pdf

[10] Seth Gilbert and Nancy Lynch. "Brewer's conjecture and the feasibility of consistent, available, partition-tolerant web services", SigAct News, June 2002.

[11] Nathan Hurst, "Nathan Hurst's Blog: Visual Guide to NoSQL Systems", http://blog.nahurst.com/visual-guide-to-nosql-systems, 2010.

[12] D.J. Abadi, "Consistency Tradeoffs in Modern Distributed Database System Design," Computer, Feb. 2012, pp. 37-42.

[13] Eric Brewer, "CAP twelve years later: How the "rules" have changed", IEEE Explore, Volume 45, Issue 2 (2012), pg. 23-29.

[14] Seth Gilbert, Nancy A. Lynch, "Perspectives on the CAP Theorem", Computer, vol.45, no. 2, pp. 30-36, Feb. 2012 